Ann. Zootech., I966, 15 (2), I35-145

\title{
ADMINISTRATION AUX ANIMAUX DOMESTIQUES D'UNE TOXINE THERMOSTABLE SÉCRÉTÉE PAR BACILLUS THURINGIENSIS BERLINER, EN VUE D'EMPÊCHER LA MULTIPLICATION DE MUSCA DOMESTICA LINNAEUS DANS LES FÈCES
}

\author{
P.-F. GALICHET \\ Station de Recherches de Lutle Biologique et de Biocconolique, \\ 78 - La Minière par Versailles ${ }^{(\mathbf{1})}$
}

\section{SOMMAIRE}

La multiplication de la Mouche domestique dans les étables pose souvent d'irritants problèmes. Cette étude a pour but de déterminer l'activité d'une substance d'origine bactérienne vis-à-vis des larves de cet insecte. Un filtrat résultant de la culture industrielle de Bacillus thuringiensis BERIIINER a été enrichi en toxine thermostable par concentration ; il contient peu de spores ; les éléments thermolabiles ont été détruits par autoclavage.

La préparation est administrée à des veaux et à des porcs, elle est incorporée à la ration au moment des repas, ou diluée dans l'eau de boisson.

Les féces excrétés par les animaux sont recueillis. Ils servent de milieux d'élevage à des larves de . Iusca domestica L. âgées de $24 \mathrm{~h}$ à $48 \mathrm{~h}$.

Des doses journatières de 50 cc à 85 cc de solution ingérée par des veaux dont le poids est compris entre roo et $400 \mathrm{~kg}$ empêchent le développement des insectes dans les fèces. La toxicité cesse $24 \mathrm{~h}$ ì $48 \mathrm{~h}$ après la fin du traitement.

Pour le porc, les doses permettant d'obtenir le même résultat sont beaucoup plus élevées (Ioo cc par jour pour des animaux de $30 \mathrm{~kg}$ ).

Sans qu'il ait été possible de déterminer avec précision les origines de cette différence, il convient d'observer que les excréments du Porc constituent un milieu beaucoup plus favorable que ceux des bovins à la croissance des larves de la Mouche domestique. L'hypothèse d'une détoxification de la matière active au cours du transit intestinal chez le Porc ne semible pas devoir être retenue.

I'ingestion par les animaux de quantités très importantes de la solution provoque une diminution de la consommation d'aliment. Étant donné la composition mal définie du produit dont nous disposions, il n'est pas possible d'attribuer ce phénomène à la toxine elle-même plutôt qu'ì l'un ou l'autre des constituants du milieu nutritif bactérien.

\section{I. - INTRODUC'TION}

La destruction des Mouches domestiques rencontre de grandes difficultés dans le milieu rural. Cet insecte, ainsi qu'un certain nombre d'autres espèces nuisibles, trouvent à l'intérieur des étables et des parcs d'élevage des biotopes propices à leur multiplication. L'abondance et la nature des gîtes larvaires constitués par les déjec-

(1) Adress: actuelle : Station de Zoologie appliquée, Bincénotique tropicale et Lutte biologique, C.R.A.A.G., Domaine Duclos, P'etit-Bourg, Guadeloupe. 
tions animales et les déchets végétaux accumulés généralement en masses importantes, rend malaisée toute intervention visant à modifier l'équilibre de la biocœenose.

Cependant, la découverte de substances qui, administrées au bétail par la voie orale sont susceptibles de traverser le tube digestif et de se retrouver intactes dans les fèces, éclaire le problème d'un jour nouveau. Ce mode de traitement doit permettre en effet d'obtenir une excellente pénétration d'un produit toxique pour les larves dans le milieu où elles se développent et d'assurer un contact précoce et intime avec les insectes à détruire. La méthode ne s'adresse pas seulement à $M u s c a$ domestica L. mais également aux autres espèces de Diptères coprophages : Musca autumnalis DE GEER, Haematobia irritans L., Stomoxys calcitrans L., Chrysomya megacephala F. ... Elle peut permettre d'intervenir également contre les Helminthes et même contre les Nématodes (CiORdia et BizzELr, I96I). Enfin, et bien que cela n'ait pu encore être vérifié pour $B$. thuringiensis B., certains produits absorbés per os ont montré une grande efficacité vis-à-vis des larves de Varron (Hypoderma bovis L., et $H$. lineatum $\mathrm{DE}$ VILLERS) dans les tissus.

Au cours de la dernière décennie, de nombreuses préparations ont été proposées aux praticiens. C'est vers I960 que les propriétés toxiques de Bacillus thuringiensis BERLINER envers la Mouche domestique ont été découvertes (HAIL et ARAKawa, I959). L'existence de cette activité antimuscide a été confirmée par la suite (DUNN, I960 Briggs, I960 ; Burns et al., I961 ; Borgatti et Guyer, I963 ; Gingrich, I965 ...).

Les préparations commerciales de $B$. thuringiensis contiennent toujours, en proportion variable, les spores du bacille et une toxine figurée protéinique, laquelle est formée pendant la sporulation de la culture bactérienne. De plus, McConner. et RICHARDS (r959) mettent en évidence l'existence d'une toxine thermostable agissant par injection dans le sang des insectes. BurgerJor et de BARJAC (Ig6o $a$ ) établissent que la souche Thuringiensis BERI,INER, sérotype I du bacille (de BARJAC et BoNNEFor, I 962) sécrète une toxine thermostable active per os vis-à-vis des larves de plusieurs ordres d'insectes. Ultérieurement BURGERJON (I962) démontre que plusieurs préparations commerciales contiennent effectivement cette toxine thermostable. Cette dernière n'a pas encore été isolée chimiquement, deux tentatives récentes en ce sens, n'ont pas abouti à des résultats concordants (CANTWELL et al., I964; de Barjac et Dedonder, I965).

La composition des spécialités mises sur le marché est donc complexe. Leur emploi est recommandé pour lutter contre divers ravageurs appartenant tous à 1'Ordre des Lépidoptères. Ces mêmes spécialités sont expérimentées sur les Diptères.

On a tendance à admettre aujourd'hui que si la mortalité chez les larves de Lépidoptères survient à la suite de l'absorption de l'une ou de l'autre des deux toxines, cette mortalité est avant tout consécutive chez les Diptères à l'ingestion du principe thermostable (BuRgerjon et de BARJAC, I962 b; CANTwEl et al., I964).

Il était donc opportun d'examiner l'activité de la toxine thermostable après séparation préalable du complexe spores et cristaux, ce dernier restant disponible pour la lutte contre les Lépidoptères. Il faut ajouter que cette séparation est souvent comprise dans le mode de récolte des spores et des cristaux tel qu'elle est pratiquée dans l'industrie. Pour celle-ci, le filtrat contenant la toxine thermostable deviendrait un sous-produit de récupération de la fabrication principale qui se trouverait ainsi valorisée. 


\section{II. - MATÉRIEL}

La préparation dont nous disposons se présente sous l'aspect d'un liquide brun foncé, plus dense que l'eau. Il a été obtenu en concentrant 24 fois le filtrat récupéré pendant la fabrication d'un produit à base du complexe cristaux-spores $\left(^{1}\right)$. Cet te solution est utilisée telle quelle dans les premiers essais, mais on la soumet par la suite à un autoclavage de 20 minutes à $20^{\circ} \mathrm{C}$.

Les animaux en expérience sont des veaux ou des porcelets maintenus constamment en stabulation. Les uns et les autres appartiennent à la Sitation de Recherches sur l'Élevage du Centre de Recherches zootechniques de Jouy-en-Josas.

L'administration de la toxine se fäit soit en l'incorporant à des granulés de farine d'orge pour les bovins, ou directement dans la ration pour les porcs, soit encore en la distribuant à la seringue ou avec un pistolet-doseur. La durée du traitement est de 7 à 14 jours. Pendant cette période et les quelques jours qui la précèdent et la suivent des échantillons sont prélevés quotidiennement ou à des dates choisies dans les déjections de chacun des animaux.

Le matériel entomologique est constitué par des larves de $M$. domestica L. souche OMS, F 33 , non résistante aux insecticides $\left({ }^{2}\right)$. Ces larves,âgées de $24 \mathrm{~h}$ à $48 \mathrm{~h}$, sont déposées au nombre de 25 par lot, dans des récipients cylindriøues contenant environ $30 \mathrm{~g}$ de fèces, clos avec une mousseline et conservés à $28-32^{\circ} \mathrm{C}$. Les excréments sont utilisés sans préparation particulière sauf en ce qui concerne les déjections des veaux nourris au lait dont il est nécessaire d'accroitre la consistance en les mélangeant à du son. L'estimation de l'efficacité d'un traitement est basée sur l'émergence des insectes adultes. Celle-ci est donc notée en fin d'expérience ; le nombre d'inagos observés est d'autant plus faible que l'action toxique aura été plus grande.

\section{III. - TECHNIQUES EXPÉRIMENTALES E'T RÉSUL'TA'TS}

\section{Essais préliminaires concernant l'efficacité et la rémanence du traitement}

Les animaux en expérience sont des veaux dont le poids varie de $50-90 \mathrm{~kg}$ pour les veaux de lait, à IIO-I $80 \mathrm{~kg}$ pour les autres.

Ils sont au nombre de 9 répartis en quatre lots :

- deux animaux témoins reçoivent une alimentation exclusivement lactée ;

- deux animaux reçoivent la même alimentation à laquelle on ajoute Io $\mathrm{cm}^{3}$ de la solution toxique à chacun des repas du matin et du soir ;

- deux animaux témoins reçoivent une alimentation solide standard (granulés d'orge, drèches, foin) ;

- les trois derniers animaux sont alimentés également avec la ration standard (5 à $8 \mathrm{~kg} /$ jour/tête), mais la solution de $B$. thuringiensis a été incorporée à la farine d'orge utilisée pour la formulation des granulés, à la dose de $21 /$ Ioo $\mathrm{kg}$ de farine. Ces granulés sont distribués à raison de I $\mathrm{kg} / \mathrm{jour} /$ tête.

Ce traitement est appliqué pendant une période de dix jours. L'essai terminé, on vérifie que les fèces ont bien perdu leur toxicité. Le produit est alors administré aux 5 animaux des deux derniers lots dans l'eau de boisson ( $10 \mathrm{~cm}^{3} /$ litre à chacun des deux repas quotidiens). Ce second essai est arrêté après sept jours.

Les résultats de ces essais sont empreints d'une certaine irrégularité : la mortalité

(1) Fourni par la Société des I aboratoires R. Bellon.

(2) Nous adressons nos remerciements à Mr le Jr P. I'ssson de l'Institut national agronomique, qui nous a assuré la foumiture de ce matériel.

Annales de Zooterhnie. - ig66. 
des insectes qui excède rarement ro $\mathrm{p}$. Ioo dans les lots témoins peut devenir élevée ( 85 p. Ioo à Ioo p. IOo) dans certains lots et rester médiocre dans d'autres. Parfois même le traitement est inopérant. Ces fluctuations paraissent indiquer que la dose choisie se trouve proche d'un seuil en dessous duquel il n'est pas possible d'obtenir un résultat satisfaisant.

Le traitement au moyen d'une préparation granulée aboutit parfois à des refus de la part de l'animal. La toxine est alors absorbée en quantité insuffisante pour que le résultat soit significatif. Ce cas mis à part, la forme solide ou liquide sous laquelle est présentée le produit n'en modifie pas l'efficacité.

Dans les circonstances favorables les excréments deviennent toxiques pour les larves de $M$. domestica L., 24 h à 48 h après l'ingestion du produit et ils cessent de l'être très rapidement après la fin du traitement. La substance toxique paraît donc accompagner les déchets alimentaires au cours du transit intestinal.

La mortalité des insectes intervient au stade larvaire ou au stade nymphal. L'examen des cadavres a révélé la présence de spores de $B$. thuringiensis $B$., mais aucun développement $\mathrm{d} u$ bacille ne s'est produit. Les spores se retrouvent d'ailleurs également dans leṣ fèces, mais rien ne permet d'attribuer la mortalité à une prolifération du microorganisme.

\section{Essais comparatits de diverses doses}

Les résultats précédents ont paru suffisamment encourageants pour que soit poursuivie l'expérimentation. Nous avons donc entrepris l'étude de l'activité insecticide de la toxine dans les excréments d'animaux auxquels on fait absorber des

TABIFAU I

Éclosion des adultes de M. domestica $L$. dont les larves se sont déreloppées dans les féces de bovins ayant ing 'ire' des doses croissantes de toxine thermostable de $\mathrm{B}$. thuringiensis 13 .

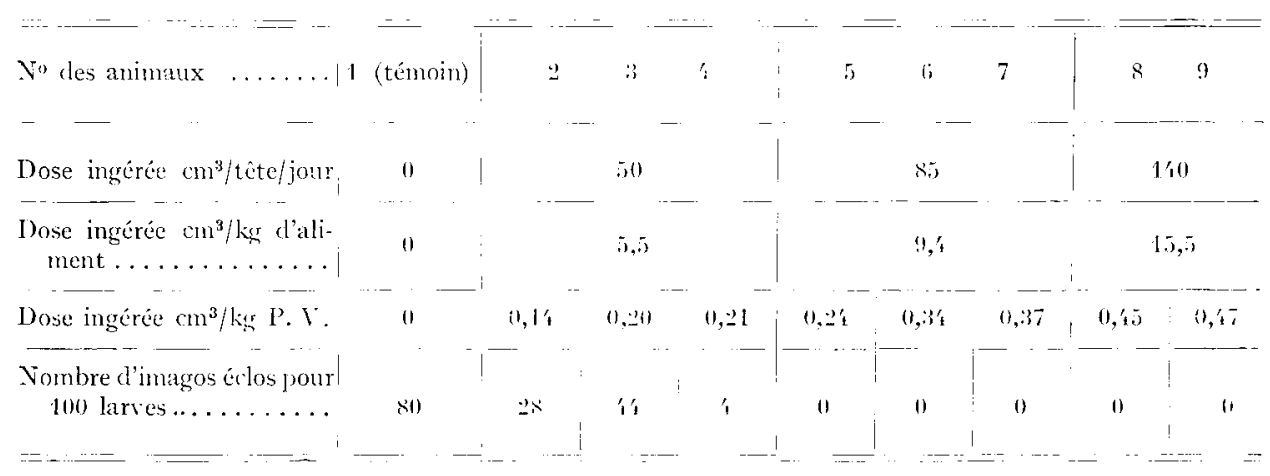

quantités variables de notre filtrat industriel concentré. Nous nous sommes limités à deux espèces domestiques : un ruminant polygastrique, le bœuf, d'une part, et un omnivore monogastrique, le porc, d'antre part. Pour des raisons pratiques, tous les essais sont effectués sur de jeunes animaux. Le poids des veaux est compris entre 200 et $380 \mathrm{~kg}$, celui des porcelets est de $30 \mathrm{~kg}$. Les premiers sont répartis 
en lots de deux à trois animaux par dose et les seconds en lots de cinq animaux. L, a solution est distribuée au veau avec un pistolet doseur tandis qu'elle est incorporée manuellement à la ration des porcelets, qui est préparée en pâté semi-liquide. Les doses étudiées sont mentionnées dans les tableaux I et 2.

\section{TABLEAU 2}

Éclosion des adultes de M. domestica $L$. dont les larves se sont développées dans les féces de Porcins ayant ingéré des doses croissantes de toxine thermostable de $\mathrm{B}$. thuringiensis $B$.

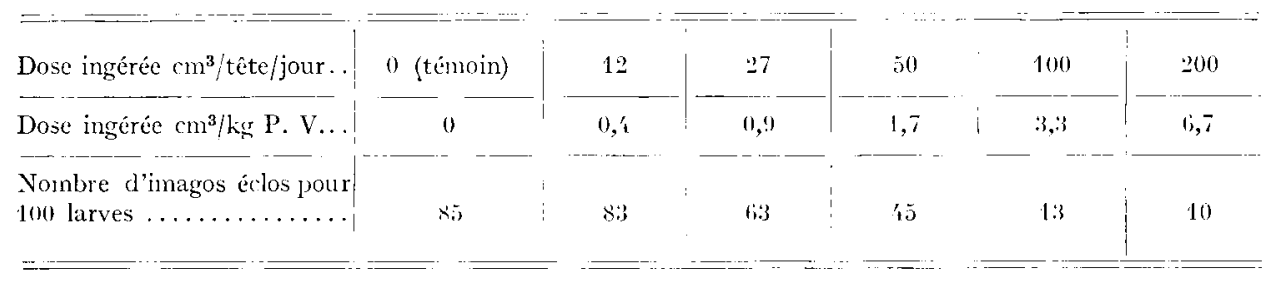

Les larves de $M$. domestica $\mathrm{L}$. sont toujours déposées au nombre de 25 par lots. Le nombre de répétitions est de 3 par traitement. Les essais sont répétés deux fois.

Les résultats sont présentés dans les tableaux I et 2 .

Pour les bovins, une dose de $50 \mathrm{~cm}^{3}$ par tête et par jour provoque une mortalité appréciable chez les larves de Mouche qui se développent dans les fèces. Ces quantités correspondent à $0,15^{-0,25} \mathrm{~cm}^{3}$ de filtrat concentré par kilo de poids vif. Aux doses plus élevées, aucun imago n'apparaît plus. Nous n'avons pas cherché à établir de relation entre l'efficacité du traitement et le poids des animaux ou la quantité d'aliment ingérée. Nous notons toutefois que pour une quantité donnée de toxine, des variations de roo $\mathrm{kg}$ (30 p. I0o) dans le poids des sujets n'ont pas eu d'influence sur la toxicité des excréments.

\section{TABLEAU 3}

Consommation journalière et variation du poids pendant la durée de l'expérience de jeunes porcelets ayant ingéré des doses croissantes de toxine thernosiable de $\mathrm{B}$. thuringiensis $B$. pendant une période de 14 jours

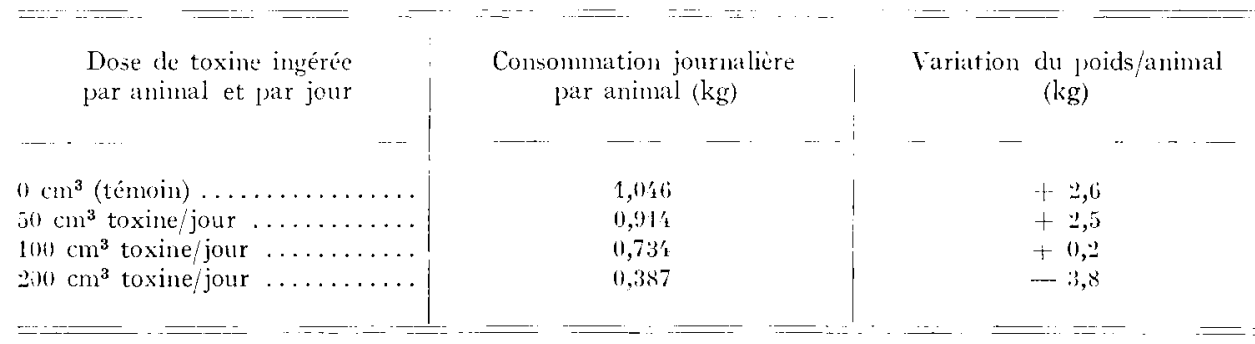

Pour le porc, les doses ont d'abord été fixées par référence à celles qui furent utilisées chez les bovins, compte tenu du poids des animaux. Il s'est rapidement avéré nécessaire de les relever d'une manière sensible : les premiers résultats favorables sont enregistrés avec une ingestion quotidienne de 50 à $100 \mathrm{~cm}^{3}$ de solution par 
animal et par jour. Rapportées au poids vif des animaux les quantités sont environ Io à 20 fois plus élevées pour les porcs que pour les bovins.

Dans le tableau 3, nous faisons figurer la consommation journalière ainsi que les variations de poids de porcelets soumis au traitement pendant une période de $\mathbf{r} 4$ jours. I a consommation décroît à mesure que la quantité de toxine distribuée augmente. En fin d'expérience, le gain de poids varie en raison inverse de la dose de produit figurant dans la ration ; la dose la plus élevée provoque même un abaissement du poids des sujets. La consommation reprend progressivement son rythme normal dans les jours qui suivent l'interruption du traitement. Notons toutefois que la plus faible dose $\left(50 \mathrm{~cm}^{3} /\right.$ jour $)$ n'apporte pas de modifications statistiquement significatives dans le comportement des animaux traités par rapport à celui des animaux témoins, compte tenu de la brièveté de l'expérimentation.

\section{IV. - DISCUSSION}

Un filtrat industriel à base de toxine thermostable s'est montré efficace contre les larves de $M$. domestica L. Ni l'absence de cristaux protéiniques, ni la réduction considérable du nombre de spores par rapport aux préparations commerciales, n'ont entraîné la perte de la toxicité. Ces résultats confirment ceux obtenus précédemment au laboratoire (Burgerjon et Galichér, I965).

L'absorption de cette préparation en quantité importante provoque parfois chez l'animal domestique le déclenchement de troubles diarrhéiques plus ou moins prononcés. Or, la larve de $M$. domestica $\mathrm{L}$. apparaît comme relativement sensible aux modifications du milieu où s'effectue son développement ; il n'est donc pas impossible qu'un affaiblissement, voire même une mortalité, puisse en résulter chez l'insecte, indépendamment de toute action toxique proprement dite. Toutefois, la similitude des symptômes observés au laboratoire par d'autres auteurs (CANTWELL et al., I964) et par nous-mêmes (GAIICHET, en préparation) avec ceux constatés dans les expériences précédentes, doit vraisemblablement permettre d'affirmer que la toxine thermostable de $B$. thuringiensis est bien l'agent principal de la mortalité enregistrée.

La comparaison quantitative de nos résultats avec ceux obtenus par d'autres n'est pas aisée : en effet, les premiers chercheurs qui font ingérer des substances à base de $B$. thuringiensis $B$. aux animaux domestiques expriment leurs résultats en fonction de la richesse en spores des préparations. Comme il a été reconnu depuis que ceux-ci interviennent peu ou pas du tout dans l'activité antimuscide, les auteurs sont amenés à préciser le mode d'obtention du produit toxique qu'ils emploient ainsi que la quantité introduite dans la ration des animaux (MECHAIAs et BEYER, I963) ou dans le milieu d'élevage des insectes (CANTWELL et al., Ig64). Il est malheureusement impossible de déterminer la teneur en matière active des substances utilisées.

Cette incertitude pourra être levée le jour où l'isolement et la définition chimique de la toxine incriminée permettront d'en faire le dosage, ou, à défaut, par l'adoption d'une méthode standard de titrage biologique par référence à un étalon déterminé (BURGERJON, I965).

D'après les auteurs, le calcul du rapport de la quantité de préparation utilisée au poids de la ration ingérée est de Io p. Ioo chez les bovins (DUNs, I960) et de 
2 à 4 p. IOO chez les poulets (BRIGGS, I960; MII,AR, I965). Cette différence devrait être attribuée selon BRIGGs à la complexité plus ou moins grande de l'appareil digestif des deux espèces. Sans doute faudrait-il aussi faire entrer en ligne de compte la physiologie de la fonction digestive.

l)ans nos expériences, nous observons un taux voisin de I p. Ioo pour les jeunes boins ; cette différence importante avec le chiffre de Duxx est sans aucun doute lié à la plus grande richesse en toxine de notre préparation. Chez les porcelets, ce rapport s'élève à ro p. Ioo ou 20 p. IOO. La résistance des larves de la Mouche domestique est donc plus élevée quand celles-ci sont placées dans les fèces de porc. Les différences de mortalité subsistent lorsque le traitement des bouses a lieu au laboratoire sans passer par l'intermédiaire de l'animal (GALICHÉr, en préparation). Il faut donc admettre, soit que les propriétés physiques ou chimiques propres aux déjections du porc diminuent l'activité de la toxine ou la rendent moins accessible aux insectes, soit que ce milieu convenant mieux à la croissance de $M$. domestica $\mathrm{L}_{\text {. }}$ (poids élevé des pupes, rapidité du développement), les individus se trouvent dans un meilleur équilibre physiologique et peuvent ainsi ingérer sans dommages de plus grandes quantités de toxine. Dans le même ordre d'idée, il est bon de noter que MEchaI,As et BEYER (I963) signalent qu'il faut diluer environ dix fois plus de produits toxiques dans les fèces que dans les milieux de cultures artificiels pour obtenir des résultats similaires.

Nous avons pu montrer au cours de nos essais préliminaires que, à doses égales, la forme liquide ou solide sous laquelle le produit est présenté, n'en modifie pas l'efficacité. Or, on n'ignore pas que la nature de l'aliment est susceptible d'influer sur les modalités du transit digestif chez les ruminants polygastriques, principalement dans leur jeune âge. Chez le veau, l'absorption de lait notamment provoque la fermeture de la gouttière osophagienne qui permet l'arrivée directe du liquide dans le feuillet ou la caillette en évitant les deux premiers réservoirs gastriques, rumen et réseau. Les aliments solides, par contre, séjournent très longuement dans ces derniers et ils y subissent les attaques de multiples fermentations microbiennes. Nos résultats montrent que l'activité insecticide de la toxine thermostable n'en est pas affectée pour autant. L'expérimentateur dispose donc apparemment d'une grande liberté pour choisir le mode de présentation le plus convenable.

Ėn ce qui concerne la rémanence, nous avons pu observer que la toxicité des fèces disparaît très vite après l'interruption du traitement, laissant présumer une élimination rapide du produit par le tube digestif des animaux. Ces résultats confirment ceux de Durir (rg60) sur les bovidés et ceux de Borgatri et Guyer (I963) sur les cailles. Le produit, par contre, persisterait longtemps dans les fumiers: MrchaLAs et BEYER font état de résultats positifs après quatre mois de stockage dans les conditions naturelles.

L'administration de préparations à base de $B$. thuringiensis $B$. au bétail ne peut être envisagée que si leur inocuité est assurée aux doses normales d'emploi. I,e traitement ne doit pas non plus affecter la valeur commerciale des animaux de quelque manière que ce soit. Cet aspect de la question a retenu l'attention des chercheurs. Les informations que nous possédons à ce sujet concernent trois espèces : les poulets, les cailles et les bovins; nos essais permettent d'y ajouter le porc.

Les premiers anteurs (Duns, rg60; BRIGGs, rg60) ne signalent tout d'abord aucun symptôme alarmant : BRIGGS notamment alimente sans danger vingt poules 
pondeuses pendant deux ans avec une ration traitée. Par la suite, on peut établir que des doses cinq à six fois supérieures à la dose normale d'emploi provoquent chez les poules une réduction de la consommation et une diminution de la ponte. MEchALAS et BEYER (I963) à qui 1'on doit cette observation, remarquent que ceci peut être simplement la conséquence du remplacement d'une fraction de la ration alimentaire normale par une substance qui convient moins bien au développement des poules. Burns et al. (I $96 \mathrm{I}$ ) signalent que la consommation des poules est plus ou moins affectée selon la préparation commerciale utilisée. Le poids des sujets et la production d'œufs sont également abaissés.

BorgatTI (I96I) montre que l'ingestion de spores de B. thuringiensis B. ne modifie en rien le métabolisme des cailles et des souris blanches. KRIEG et HERFS (rg63) confirment que ces dernières peuvent absorber, par voie orale ou parentérale des quantités variables de surnageant d'une culture de $B$. thuringiensis var. thuringiensis sans présenter de réactions défavorables.

Nous avons nous-même procédé à des essais préliminaires sur des souris blanches d'un poids moyen de $30 \mathrm{~g}$. Nous avons injecté dans la cavité abdominale différentes doses d'une solution à Io p. Ioo de notre filtrat industriel. Nous n'avons pas enregistré de mortalité aux doses égales ou inférieures à $0,05 \mathrm{~cm}^{3}$ de filtrat. Cette dose équivaudrait pour un bovin de $300 \mathrm{~kg}$ à un volume de $500 \mathrm{~cm}^{3}$. Il semble donc que nous disposions d'une marge de sécurité suffisante pour l'utilisation de la solution.

L'administration pey os aux bovins a montré que les quantités de $50 \mathrm{~cm}^{3}$ à $85 \mathrm{~cm}^{3}$ par jour sont bien tolérées. Au-delà ( $140 \mathrm{~cm}^{3}$ jour), nous observons, après quelques jours, une perte d'appétit chez les animaux. Nous faisons la même constatation chez les porcs à qui on fait absorber quotidiennement roo à $200 \mathrm{~cm}^{3}$ de la solution. L'interruption du traitement permet à l'animal de retrouver rapidement son équilibre. Le produit utilisé n'étant pas purifié, il n'est pas possible d'attribuer les troubles observés à la toxine plutôt qu'à l'un ou l'autre des constituants du bouillon de culture microbienne. De nouveaux essais sont donc indispensables.

Il avait été envisagé de distribuer à un lot d'animaux témoins, un filtrat d'une culture de $B$. thuringiensis dont la toxine thermostable soluble serait absente soit qu'elle ait été détruite par un procédé quelconque, soit que 1'on aie pris soin d'utiliser une souche de bacille ne produisant pas cette toxine. Les difficultés techniques auxquelles nous nous sommes heurtées pour obtenir des milieux comparables avec ou sans toxine nous ont fait renoncer à ce projet.

Le problème de la toxicité vis-à-vis de l'homme, des vertébrés, et aussi de la faune auxiliaire, de produits bactériens doués d'une activité pathogène envers les insectes nuisibles, a déjà été étudié dans le cadre général de la lutte biologique. C'est ainsi qu'il a été démontré à maintes reprises que la plupart des organismes entomopathozènes font preuve d'une certaine spécificité d'action. Bien que dans ce domaine les bactéries du groupe Thuringiensis se comportent d'une manière plutôt moins sélective que d'autres, leur utilisation a néanmoins peu d'effets sur un grand nombre d'insectes entomophages. Cette sélectivité renforce l'intérêt pratique de ces substances.

A propos des animaux à sang chaud, Brown (in STEInhaus, I959) écrit : " Il est difficile, sur des bases génétiques, d'imaginer qu'un microorganisme entomopathogène puisse être simultanément pathogène pour les Vertébrés ", et STFINHAus (I959) ajoute le commentaire suivant: "Dans les conditions naturelles, B. thurin- 
giensis n'est jamais associé avec des Vertébrés, même sous un état non pathogène ", puis plus loin "Bien qu'il ne soit pas impossible que les animaux ingèrent un feuillage traité avec des spores de $B$. thuringiensis, il n'existe aucune raison de penser qu'à la suite d'un tel contact une bactérie puisse s'établir dans le tube digestif des animaux, ni que ce contact, aussi intime soit-il, ait plus de chance d'aboutir à une infection que dans le cas des commensaux qui composent la flore intestinale. "

Dans ce domaine, les essais auxquels nous nous sommes livrés sur porcs et bovins peuvent fournir d'utiles indications sur les dangers que font courir au bétail et au gibier les dépôts résiduels de toxine thermostable consécutifs aux applications insecticides effectuées sur les cultures ou les forêts. Des considérations théoriques et des démonstrations expérimentales amènent BROWN et STELNHAUs à répondre négativement à la question de savoir si des formes pathogènes pour les Vertébrés ne pourraient pas apparaître par mutation spontanée de souches entomopathogènes.

Rappelons d'ailleurs que l'emploi des substances d'origine microbienne est banal en thérapeutique vétérinaire et humaine depuis la découverte des antibiotiques. Ces derniers sont même passés dans la pratique zootechnique elle-même, certains d'entre eux (Auréomycine, Érythromycine) étant utilisés par les éleveurs comme facteur de croissance. Des levures diverses (Saccharomyces spp.) entrent depuis longtemps dans la composition d'aliments pour le bétail en raison de leur intérêt alimentaire et de leurs propriétés appétitives (SALMoN-LEGAGNEUR, Ig60). Dans ce domaine, l'introduction de poudre à base de $B$. thuringiensis $B$. ne constituerait donc pas une innovation.

S'il paraît exclu que les souches entomopathogènes provoquent des accidents toxémiques ou septicémiques chez les Vertébrés, il n'est toutefois pas impossible que les toxines sécrétées par certaines d'entre elles provoquent des réactions allergiques chez quelques sujets. La plupart des substances de nature protéique sont d'ailleurs dans ce cas. Il serait rencontré chez 1'Homme avec un champignon, Beauveria bassiana BALs.

Enfin, il peut être rappelé, à propos plus particulièrement de la toxine thermostable de $B$. thuringiensis B., que la mortalité des insectes survient le plus souvent à l'occasion d'un changement de stade larvaire ou à la métamorphose, phénomènes développés surtout dans le monde des Arthropodes.

Vues sous un angle toxicologique, les perspectives d'utilisation de $B$. thuringiensis en médecine vétérinaire se présenteraient donc sous un jour favorable.

\section{V. - CONCLUSION}

La lutte qu'il est souvent nécessaire d'entreprendre contre les pullulations de la Mouche domestique associe généralement les mesures d'hygiène et l'emploi de substances toxiques.

Les difficultés rencontrées pour obtenir la pénétration d'un insecticide dans la masse de déchets organiques où se développent les larves de $M$. domestica L. peuvent être surmontées par l'adjonction à la ration d'un produit qui, traversant sans subir d'altérations le tube digestif des Vertébrés, se retrouve dans les fèces. La préparation doit être inoffensive pour l'animal domestique et active contre les larves de l'insecte. 
Différents auteurs ont déjà utilisé dans ce but des préparations hétérogènes renfermant des spores de $B$. thuringiensis $B$. Ils ont appliqué ce mode de traitement soit à des mammifères, soit à des oiseaux.

Nous avons pu obtenir des résultats positifs avec une solution enrichie en toxine thermostable, pauvre en spores et dont tous les éléments thermolabiles ont été éliminés. Cependant, nous avons montré que de grandes différences d'efficacité peuvent apparaître d'une espèce à une autre et qu'en conséquence une mise au point est nécessaire pour chacune d'elles.

La recherche entreprise n'avait pas pour objet essentiel la découverte d'un remède aux pullulations des Mouches dans les étables, malgré l'importance de ce problème d'hygiène publique ; il peut exister d'autres méthodes plus efficaces ou plus économiques que celles que nous envisageons ici. Toutefois celle-ci présente certains caractères propres susceptibles d'en développer l'intérêt théorique si on la replace dans le cadre général de la lutte biologique au moyen des germes.

Cette dernière a été exclusivement considérée sous l'aspect des mesures de protection phytosanitaire : elle n'a jamais été appliquée, à notre connaissance, contre des insectes d'importance médicale ou vétérinaire. Cette voie nouvelle, abordée en observant toutes les règles de prudence que recommande P. GRIsox (Ig6r) lorsque 1'Homme veut juguler l'action néfaste de ses ennemis, ne devrait pas se révéler moins féconde que la première.

Reç pour publication en mars 1966.

\section{VI. - REMERCIEMENTS}

Nous remercions nos collègues R. Jarrige, C. Mathiet, A. Rérat el E. Salmon-Legaginetr de la Station de Recherches sur l'Élevage, C.X.R.Z., qui ont bien voulu mettre à notre disposition les animaux nécessaires à nos expériences.

Nous remercions également MM. Y. MANis, ingénieur à la Station de Recherches sur les Ruminants et R. Merte, agent technique, ainsi que MM. A.-M. Morsant et J.-P. Tero de la Station d'Élevage des Porcs de la Minière pour l'aide que les uns et les autres nous ont apportée à la réalisation matérielle des expériences.

\section{SUMMARY}

PREVENTION OF REPRODUCTION OF " MUSCA DOMESTICA " IN FECES BY FEEDING WITH A IHETT-STABLE TOXIN FRON " BATLLLS THIRINGIEXSIS " BERLINER

A filtrate, highly concentrated in heat-stable $10 x i n$ and much less in viable spores, and whose heat-labile elements were destructed by autoclaving, was obtained from commercial formulation of Bacillus thuringiensis BerLINER and given to calves and piglets in different forms : for calves, it wats either lirpuid or solid by incorporating it to granulated barley-meals; for pigs, the tiltrate was added to the semilicuid mash of the ratio.

The feces of tested animals were collected and served as a mediun of culture of house-fly larvae whose mortality was watched over. It was thus establisherl that 50 to 85 or ingested daily by calves from 100 to $400 \mathrm{~kg}$ weight prevent the insect from developing in feces. Toxicity ended up 24 to 48 hours after the treatment was stopped.

In the Pig, much greater amounts (I Io cc daily for $30 \mathrm{~kg}$ piglets) were refuired in order to obtain similar results. It should be noticed however that the P'ig's fecae is a mediun much more suitable for the development of house-lly larvae than the Calf's (higher weight of pupae, shortened larval stage). Investigations in progress in our laboratory enabled us to find the very same difference when feces of both species are treated in vilro without the filtrute being transported through the alimentary canal. 

form.

The efficiency of the treatment was the same whether the filtrate was given in solid or liquid

Insects sometimes died as larvae, but death is most frequently delayed until nymphal stage. This is typical of the heat-stable toxin ; so are the malformations observed in either pupa or imago (CANTwELL et al., I964). Fxamination of dead larvae revealed the presence of Bacillus thuringiensis spores, but no development of them : therefore, nothing entitles us to think that mortality is due to a proliferation of the micro-organism.

The reactions of the animals to the treatment was studied on pigs. We noticed an anorexia which may be important when the daily rate is high, and a consequential loss of weight at the end of 1 he experiment. The animals recover soon after the treatment is ended. But since the composition of our commercial product is not clearly determined, there is no reason why we should attribute the disturbance observed to the toxin rather than to any other constituent of the nutritive bacterial medium.

Further investigations are thus recpuired.

\section{RÉFÉRENCES BIBLIOGRAPHIQUES}

Borgater A. L., (iuykr (3. E., I963. The effectiveness of commercial formulations of Bicillus thuringiensis BERLINER on house-fly larvae. J. Insed. Pathol.. 5, 377-84.

BRiggs J. D., rg60. Redution of adult house-fly energence by the effects of Bacillus spp on the development of immature forms. I. Insecl. Pathol., 2, 4 is-43t.

Burgerjon A., de Bar fac II., $1960(a)$. Nouvelles domées sur le rôle de la toxine soluble thermostable produite par Bacillus thuringiensis BERLINER. C. R. Acal. Sci., 251, 9I1-9I 2.

Burgerjon A., ig6z (b). Principes themostables dans les préparations industrielles à base rle Bacillus Iluringiensis berLixer. Coll. Inler. Pathol. Insecles, Paris, 227-237.

Blakgerjon A., 1965. Au sujet de la carastérisation des produits à base de Barillus thuringiensis BERLINER par rapport au titrage biologique de la toxine soluble thermostable. Entomophaga, 10, $55-65$.

Burcierjon A., (ialichet P. F., 1965. The effectiveness of the heat-stable toxin of Bacillus thuringiensis var. thuringiensis BERLINER on larrae of Mlasca domestica Lixidets J. Invert. Pathol., 7, 263-264.

Burns F. C., Wilson 13. II., Tower B. A., ighi. Eiffect of feeding Bactllus thuringiensis to caged layers for fly control. J. Econ. Entomol., 54, 913-915.

Cantwer G. E., Henpel A. M., Thompon M. J., 196. 'The production of an exotoxin by rarious crystal-forming bacteria related to Bacillus thumginsis var. Muringiensis berLiner I. Inse't. I'athol, 6, $466-480$.

Corda H., Bizzet W. E., 1965. A preliminary rejort on the effects of Bacillas Ihumingiensis vatr. Tharingiensis BERLINER on the developjement of the free living stages of some cattle nematorles. I. Parasitol.,47, 4I.

De BarJac If., Bonnerom A., inge. Essat de classification biochinique et serolorique te 2 souches de Bacillas du type Bacillas thuringiensis. Enomophaga, 7, 5-32.

De Bariac II., Dedonier R., 5965. Isolement d'un nucleotide identifiable it la "toxine themostable "

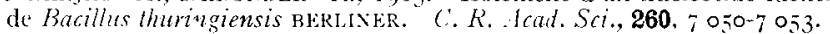

DUNx I'. H., I960. Control of house flies in bovine feres by a feed additive containing Bacillus lhumingensis var. tharingiensis BerLixER /. Insecl. Palhol., 2. I3-16.

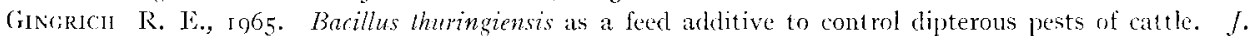
Econ. Iintomol., 58, 36,3-364.

Grison P., rgor. T'utilisation d'une bactérie Bucillus thurngiensis clans la lute biologique contre les in-

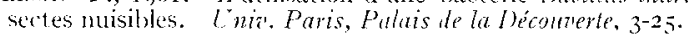

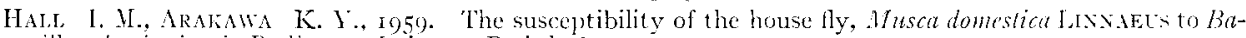
cillus tharingiensis Berlinet. J. Insed. Pahol., 1, 351-35.

KRIEG A., HeRFs W., 1963. Empfindlickkeit verschiedener hocktenarten gegenüber rlen " Exotoxin " som Bacillus thuringiensis BERLIXER. Pfansenkrankh. Pfanenschus, 70, I 1-21.

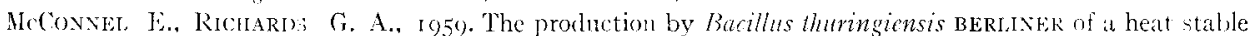
substance toxic for inserts. Canad. J. Ticrobiol., 5, i61-168.

Mechalas I3. J., Beyer O., 1963. Production and assay of extracellular toxins by Bacillus thurineiensis. Developments in indastial microbiol.,4.1+2-147.

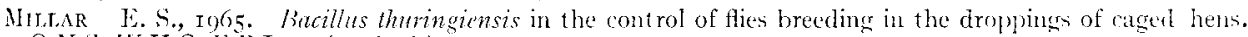
O MS. WHO. H B I to (ronétés) + J.

Salmon-Legagneur E., igGo. Jes préférences alimentaires du porcelet. IV. Appétibilité des levares. Anm. Zoolech, 3, $255^{-260}$.

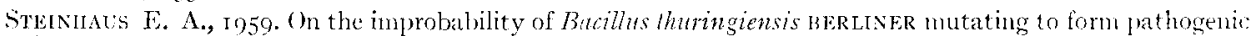
for vertebrates. J. Econ. Entomol. 52, 506-508. 